\title{
La Flexibilidad Laboral
}

Fausto Miguélez Lobo *

RESUMEN:

La flexibilidad del trabajo es boy la gran estrategia de las empresas frente a una competitividad mucbo más global que hace décadas. Las incertidumbres se ban acumulado sobre el mercado y la tecnologia -factores no tan fáciles de controlar y mantener como antes- lo que ba llevado a las empresas a centrar sus certidumbres sobre lo que mejor pueden controlar localmente, el factor trabajo. Como consecuencia, esta disponibilidad requerida en boras de trabajo, salarios, contratos, que llamamos flexibilidad, se convierte en incertidumbre para los trabajadores. La más evidente está en la inestabilidad de sus empleos.

Por ello la estrategia de flexibilidad tiene dos caras: una económica la otra política. En términos económicos la flexibilidad permite controlar mejor los costes de la producción, mientras que en términos políticos lo que se consigue es controlar mejor las demandas de los trabajadores y de sus organizaciones.

\section{Palabras Claves:}

Flexibilidad, Relaciones Laborales, Norma social del empleo, Estrategias de flexibilidad, tiempo de trabajo, cohesion social.

- Catedrático de Sociología. Universidad Autónoma de Barcelona • fausto.mígueles@uab.es 
ABSTRACT:

Work flexibility is today the most important strategy of companies in front of a competition much more global as it was decades ago. Uncertainties acumulate on market and technology -as factors more difficult to be controled as in the past-what is the reason to companies to move control and new certainties over the third factor they manage localy, the work. As a consequence this demanded availability in bours, salaries, contracts, called flexibility, turns into uncertainty to workers, being most evident as instability of employment.

This is the reason why flexibility is a double-faced strategy. In economic terms flexibility allows to controle better costs of production whereas in political terms it controles demands from workers and their organisations.

KEY WORDS:

Flexibility, Industrial Relations, Social Rule of Employment, Working Time. Employment strategies, Social Cobesion

\section{INTRODUCCIÓN}

La flexibilidad laboral es una de las respuestas más importantes que las empresas están dando a los cambios que han tenido lugar en la organización del trabajo y en el mercado, sea en el ámbito nacional que global, en los últimos años. Pero además de esto, desde hace casi tres décadas domina el discurso de la flexibilidad (Pollert, 91). Se dice que el mercado de bienes y servicios se ha flexibilizado en el sentido de que es menos estándar y se ha tenido que especializar según demandas más particulares, lo que ha llevado a la flexibilización -es decir, una utilización más diversificada- de la tecnología y del trabajo y éstas, necesariamente, a la del mercado de trabajo y del empleo. El razonamiento es muy discutible y particularmente lo es el supuesto determinismo que se asume en la deducción mencionada. Cuestionar tal determinismo será uno de los objetivos de este artículo.

Para empezar no es tan seguro que el origen del proceso esté, o esté siempre, en demandas más especializadas -es decir, en los clientes y sus necesidades o deseos- sino que puede estar en la imposición de ciertas tecnologías en vez de otras por los intereses de los fabricantes o de quienes controlan la energía, o bien puede referirse a las estrategias de venta de unos y otros. O también podría ser la decisión empresarial de "producir necesidades diferenciadas" por las connotaciones de distinción social que ello pueda conllevar, por lo que en este caso simplemente lo que originaría el proceso serían estrategias de venta del producto. Pero también podría ser que las cosas empezaran justamente por el extremo opuesto: es decir, que el origen del proceso podría estar directamente en una estrategia de flexibilización del mercado de trabajo y del empleo con el ánimo de reducir los costes del trabajo o de introducir criterios más ágiles y más fáciles de control del 
mismo. Este es mi particular punto de vista. En cualquiera de las alternativas señaladas o en la combinación de varias de ellas se quiebra la supuesta lógica de que se trata de "respuesta a nuevas demandas" y pueden aparecer en el tema aspectos que son claramente ideológicos, en el sentido de que responden a una construcción artificial de una realidad para justificar prácticas -en este caso prácticas empresariales- que nada tienen que ver con dicha lógica.

Lo que, en todo caso, es evidente, es que estamos inmersos en estrategias de flexibilización del mercado de trabajo y del empleo que son presentadas como mera consecuencia de cambios anteriores cuando no está demostrado que haya de ser así. En realidad se trata de la utilización flexible en lo que respecta a contratación, tiempo de trabajo y condiciones de trabajo, llegando a la des-contratación temporal o definitiva cuando convenga. Como si de un proceso histórico inapelable se tratase, se suele agregar que el trabajo y el empleo estables son fenómenos de la primera fase del capitalismo, mientras que en el momento actual ha de predominar la flexibilidad porque la realidad y la vida en su totalidad serían flexibles.

Rigurosamente hablando no siempre y necesariamente se está dando un mercado de bienes y servicios más flexible y, cuando se da, no necesariamente se deben seguir de ahí las otras flexibilidades. Respecto al segundo punto, volveremos posteriormente. En cuanto al primero es necesario señalar los tintes de exageración que se registraron en las primeras reflexiones sobre la "producción flexible" a resultas de la obra de PioreSabel (1990), puesto que con más frecuencia lo que se ha dado no han sido productos y servicios a la carta, sino un producto o servicio básico con ciertas variaciones a la carta. Caso típico es el automóvil, que sigue siendo montado en cadena, pero con la posibilidad de introducir variantes notables en un mismo modelo. De todos modos, cabría pensar que a exigencias flexibles del mercado -más o menos productos, productos de un tipo o de otro, servidos con mayor o menor rapidez- se puede responder a veces con más o menos flexibilidad de la tecnología y de la organización, sin tocar el empleo. Otras veces se puede agregar a lo anterior la flexibilización pactada del tiempo de trabajo, sin llegar a flexibilizaciones del mercado de trabajo que puedan afectar a las condiciones de vida de los trabajadores. Y hay casos -típicos en sectores como el comercio (rebajas o ventas de temporada) o la hostelería (puntas de turismo)- en los que necesariamente la flexibilidad tendrá que afectar al mercado de trabajo, cosa que se puede hacer en formas diferentes: con más o menos garantías de futuro en el momento del cese de la ocupación (Alós R. y otros 1988). Por ello sería conveniente que se lograra discemir lo que sencillamente son ideologías de flexibilización ("flexibilizar es bueno", "quien no se flexibiliza desaparece") que ocultan estrategias y objetivos interesados de lo que son estrategias de flexibilización que pueden pactar empresas y sindicatos o trabajadores individuales, y lo que son procesos materiales que se han hecho más flexibles, es decir, que han introducido mayores posibilidades de substitución de unos materiales por otros o de una organización por otra. 
Cierto es que algunos mercados se han hecho más flexibles en el sentido de requerir bienes y servicios más a la medida de los clientes, aunque en muchos otros casos los bienes y servicios siguen siendo estándar o bien sobre la base estándar se pueden introducir modulaciones para satisfacción de demandas particulares. Pero otra cosa bien diferente es que muchos mercados se han hecho inestables, porque la competencia es más fuerte o el apoyo de los Estados es menor o ha cambiado de orientación. El resultado es que las empresas pueden tener muchas más incertidumbres que en el pasado por lo que al mercado se refiere: tienen que conquistarlo y mantenerlo cada día. no pueden dormirse sobre certidumbres supuestamente adquiridas para siempre.

Parece evidente que las empresas han asumido estrategias de flexibilidad para hacer frente a incertidumbres que hoy tienen y no tenian, o tenian en menor grado, en el pasado. Contra la incertidumbre del mercado - ¿lograr vender o no?- se adopta la flexibilización en la elaboración de bienes y servicios. Contra la incertidumbre de los stocks se incrementa la flexibilidad de la organización del trabajo, la más evidente la de "just in time". Contra la incertidumbre de los costes del trabajo, la posibilidad de utilizar éste en mayor o menor medida, en unas u otras categorias. Frente a la incertidumbre de la fuerza del otro actor económico, el trabajo, puede ser adecuada una estrategia en que aquella tenga una base muy insegura (muy precaria). En una palabra, la flexibilidad es una estrategia de las empresas, que con ella se garantizan medios y caminos alternativos a los tradicionales. "Flexibilizar el factor trabajo" implica ciertamente aspectos técnico-organizativos, pero también intereses diferenciados, quizá incremento de desigualdades, pérdidas y ganancias, es decir. estamos hablando de un tema que no es meramente técnico, sino que tiene implicaciones muy claras en intereses económicos y de poder. Es obvio que, por ello, los actores sociales desarrollen estrategias en torno a la flexibilidad. Es necesario entender a fondo la flexibilidad para comprender esas posibles estrategias diferenciadas o hasta contrapuestas. Analizar los vínculos entre flexibilidad laboral y nuevas estrategias empresariales en el trabajo, así como las respuestas de los trabajadores o de sus representantes, es otro objetivo principal de estas páginas.

\section{La Norma soclal del empleo del Estado del Bienestar}

Para ello es conveniente refirirse a dos realidades, distintas aunque profundamente interconectadas, el trabajo y el empleo. El trabajo es la actividad.por la que los humanos, con la ayuda de instrumentos cada vez más sofisticados, transforman la naturaleza, los materiales y la tecnología misma con el objetivo de producir bienes y servicios que satisfagan sus necesidades y deseos. No entramos ahora a discutir sobre quién decide esas necesidades y deseos. El empleo es la forma de relación social que adquiere dicha actividad, con otras palabras el papel social que cada 
persona asume en dicha actividad, yendo aparejadas a ese papel unas condiciones materiales y sociales, una posición social, unas formas de entender dicha actividad. unas formas culturales y una simbología (Watson, 1994). La flexibilidad puede referirse al trabajo y al empleo.

Las transformaciones en el trabajo tienen importancia en términos sociales y sociológicos, puesto que el tipo de trabajo y las formas en que se trabaja influyen no poco en la categoría social de las personas, aunque menos que en el pasado según ciertos autores (Offe,1991). Pero las transformaciones del empleo son de mucho mayor calado, puesto que no sólo definen en mayor profundidad la identidad social (Alonso, 2000), sino que también condicionan la vida cotidiana en el trabajo. Por ello lo que más interesa aquí es que nos centremos en lo que se llama la flexibilidad en el empleo.

Diversos autores interpretan el papel que los trabajadores juegan en la gran transformación socio-económica que tiene lugar en Europa descle los años 40 en adelante -principalmente su participación en el estado del Estado del bienestar-con una referencia explícita al empleo. Señalan que se configura en esos años una nueva norma social del empleo (Prieto, 2002; Castel, 1995). No se trata de una norma jurídica aunque tiene apoyos jurídicos, sino de una norma social, es decir, un "acuerdo", un equilibrio o un "statu quo" que se asume como normal. Consiste en que los ciudadanos que así lo desean pueden convertirse en ciudadanos laboralmente activos, con un contrato -socialmente hablando, un estatus reconocido- estable en su trabajo. Ese contrato estable les permite obtener unos ingresos estables con los que programar sus vidas en la perspectiva de años. Por supuesto no es una concesión gratuita ni momentanea, sino que dicha situación de empléo se consigue tras años de luchas colectivas. Ni es una situación estática, sino que los trabajadores, en general a través de sus representantes, negocian y mantienen cada año su poder adquisitivo, mejoran sus condiciones de trabajo, reducen el tiempo de trabajo. Pero ello tiene repercusiones inmediatas sobre el trabajo en términos de promoción, mejora de la autonomía y en muchos casos de la participación.

En resumen, ese modelo de empleo es el empleo estable. Aunque ésa no sea la situación de todos los trabajadores, sí es la normal, aquella a la que razonablemente se puede aspirar. Aquella que en bastantes casos parece vinculada con la formación recibida previamente a entrar en el mercado laboral. En términos intergeneracionales esa norma de estabilidad no sólo permite que los hijos puedan mejorar la estela de los padres sino mejorar respecto a aquellos. La gran transformación de los años 80 es que esta norma social se empieza a resquebrajar o en ciertos países directamente se rompe. $Y$ ese debilitamiento no tiene que ver sólo con las transformaciones en las empresas y en el trabajo, sino con contextos económicos y políticos mucho más generales (Míguelez-Prieto, 2002), aquellos que habían sido su origen, lo que nos viene a indicar que también en dichos contextos debemos 
buscar la explicación del cambio, aunque sin olvidar la referencia de las empresas.

Pero para ser realistas en la apreciación de los fenómenos que estamos analizando, conviene no olvidar dos cuestiones fundamentales. La primera es que esta norma social del empleo descrita ha estado vigente en unos pocos países del planeta, y aún con diferencias a veces bastante notables entre ellos, siendo su vigencia de pocos años. La segunda es que básicamente se refería a los hombres y no a las mujeres después del matrimonio. Por tanto nos estamos refiriendo a un fenómeno, a una forma de trabajar, a unas relaciones sociales basadas en el trabajo que más bien son la excepción de lo que ese aspecto de la vida ha sido para la mayoría de los habitantes del planeta Tierra. Cabe preguntarse porque están cambiando las cosas es estos países, pero sin olvidar que es más aún más importante la otra pregunta: ¿por qué nunca han cambiado en el resto?

\section{Flexibilidad en el proceso de producción y de trabajo}

En los años 70 y 80 se registra una cierta crisis en las formas dominantes de producir, esto es, en el taylorismo-fordismo, porque éste resulta rígido en exceso para las demandas que provienen del mercado (Piore-Sabel, 1984). Se dice que se ha estancado la producción en grandes series porque no hay suficiente demanda de las mismas en los países centrales -en algún grado están saturados de ese tipo de bienes- sin que se hayan abierto nuevos mercados en otros países. La realidad más bien es que la crisis proviene de la bajada de la demanda, que se produce por el aumento de la inflación y la disminución de los ingresos derivada de la crisis del empleo. Por el contrario, se mantendría la demanda de quienes buscan diferencia y distinción y que siguen teniendo un gran poder adquisitivo. Por ello la rígida organización del trabajo taylorista-fordista debe ser retocada para ir a producciones más "a la carta", más cambiantes y flexibles y en general más cortas. No cabría olvidar que poner el acento en la diferencia y la distinción pueden ser también una forma de vender, no necesariamente el reflejo del deseo de los consumidores.

Una consecuencia obvia de esto parece ser la necesidad de una organización del trabajo más flexible. Esto hace referencia a la forma en la que se ejecuta el trabajo, al control que sobre el mismo tiene el trabajador, a la relación entre trabajo y capacidades de formación, a la satisfacción en el trabajo, a las condiciones físicas y mentales en las que se realiza el trabajo y a un largo etcétera. Esa organización del trabajo más flexible puede dar paso a trabajos más enriquecidos (Kern-Schuman, 1988) o a trabajos más empobrecidos (Miguélez,Alós,Recio, 1988). Dicho con otras palabras, la necesidad de realizar producciones más "personalizadas" de bienes y servicios puede dar origen a un mayor control del trabajador sobre las diversas fases de su trabajo, porque le permite introducir "microdecisiones" en fases determinadas del proceso. Es una consecuencia positiva de la flexibilización. Pero tam- 
bién puede originar trabajos empobrecidos, en el supuesto que la utilización de ciertas tecnologías permite la apropiación por la organización de conocimientos que, desposeídos de sus propietarios, pueden ser aplicados también a producciones más cortas y más personalizadas. Todo dependerá de las estrategias que acaben dominando en el proceso productivo.

A mi entender hay, sin embargo, razones más contundentes que las hasta ahora mencionadas. En realidad las empresas necesitan recuperar el control sobre una organización del trabajo que, en el periodo más netamente taylorista-fordista, estaba muy normatizada $y$, por tanto, dejaba en manos de los sindicatos, de los equipos de trabajo o de los trabajadores parcelas importantes de actuación y hasta de decisión (Burawoy, 1989), al tener la dirección que respetar las normas de la organización establecidas. A mi entender éstas son las razones más contundentes del cambio que está teniendo lugar, puesto que la flexibilidad del trabajo, y más aún la flexibilidad del empleo, en realidad lo que permite a las empresas es retomar un mayor control sobre la organización del trabajo. Los cambios hacia una organización del trabajo mâs flexible no siempre se están obteniendo por imposición (Quit, 1997), sino también por negociación. Pero con frecuencia dicha negociación la empresa no la realiza con los sindicatos, sino con grupos de trabajadores o con trabajadores individualmente lo que hace dudar de si esta negociación se debe entender como el contraste entre propuestas que es lo habitual o como una imposición solapada.

Tanto los autores llamados institucionalistas como los regulacionistas (Boyer, 1986; Aglietta, 1987) coinciden en que los años 80 suponen una fuerte crisis de las formas de producir tradicionales en los países capitalistas. Quienes miran los cambios desde la perspectiva de los productos y del mercado hablan de producción flexible; quienes lo hacen desde la perspectiva de la organización del trabajo prefieren hablar de neo-fordismo. La producción flexible se nos presenta desde dos teorías que son complementarias: por un lado la de la reducción de la demanda que llevaría a recortar la producción en serie y poner el acento sobre la calidad con el objetivo de reactivar dicha demanda; por otro lado estaría la teoría de la diversificación de la demanda que tendría en cuenta la existencia de bienes diferenciados y no de masas, es decir, implicaría la especialización de los mercados. En ambos casos los autores conceden a los consumidores o clientes una importancia determinante en los cambios, como si el mercado estuviese dinamizado por la demanda de bienes y servicios, de acuerdo a una supuesta racionalidad según la cual los humanos transformarían la naturaleza para hacer frente a sus necesidades y deseos. Pero dicha racionalidad es más que dudosa en el sistema de producción (Polanyi, 1989). Si no tuviésemos otros argumentos, hoy día el gran deterioro medioambiental sería razón suficiente para entender que ni siquiera hay una racionalidad económica. 
Mi punto de vista, por el contrario, es que ya hablemos de producción flexible ya de neo-fordismo deberíamos hacerlo principalmente desde la perspectiva de las empresas y, por tanto, las preguntas pertinentes tendrian que ver con el cambio de estrategias empresariales. Si consideramos que no se trata tanto de especialización de la demanda o de diferenciación de gustos, sino de que ha crecido la competitividad en un mercado que anteriormente podía ser menos competitivo porque estaba más protegido o sencillamente porque había menos empresas o bien porque éstas tenían más posibilidades de control, lo que corresponde ahora es justamente examinar los cambios en las estrategias de las empresas para hacer frente a los nuevos retos de la competitividad. Podemos observar que hay una multiplicidad de estrategias empresariales, todas ellas dirigidas a reducir los costes de la producción de bienes o servicios desde diversas aproximaciones.

En primer lugar tenemos estrategias de internacionalización de la actividad empresarial que permiten dos objetivos: el primero es captar nuevos mercados desde dentro (los de los países en que las empresas centrales establecen sus empresas filiales o asociadas), el segundo es producir bienes o componente o partes de servicios con menores costes laborales en dichos países, siendo luego trasladados esos productos a países de mayor capacidad adquisitiva en los que son montados en bienes finales o en procesos de servicios más complejos (Castells, 1997). Una parte de esos bienes pueden ser elementos estándar a los que se referían algunos de los autores mencionados que pueden ser montados en productos finales que tendrán una presentación más personalizada. Dentro de un mismo país podemos tener diversos procesos de descentralización y subcontratación. Las empresas subcontratan o descentralizan productos, componentes, servicios que resulten costosos de mantener integralmente, asumiéndolos en el montaje final o por vía de servicio externo o simplemente por vía comercial. Es también una forma de reducir costes, puesto que en otros sectores económicos, en empresas más pequeñas, en otros territorios del mismo país determinados costes, particularmente los de la mano de obra, pueden ser menores.

También se da, por supuesto, la especialización de empresas -0 de líneas de producción o servicios dentro de la empresa- para mercados más reducidos, más especializados o más personalizados. Todas estas estrategias son flexibilizadoras en el sentido de que eliminan incertidumbres de diverso tipo a las empresas: de mercado, de aplicación de la tecnología, de costes del trabajo, de tasas de diverso tipo. Por tanto la iniciativa de las mismas está en las empresas, aunque ciertamente el mercado es el acicate por cuanto puede exigir una competitividad mucho más compleja.

La flexibilidad tal como la hemos descrito hasta ahora es la cara técnica de la lucha contra las incertidumbres que suscitan las formas tradicionales de actividad económica. Sin embargo detrás de esta cara técnica hay unos aspectos sociopolíti- 
cos que son aquellos que más nos pueden interesar desde la perspectiva en que ahora los analizamos porque son los que mejor pueden explicar estrategias vinculadas a intereses. Las empresas han tenido que conjugar siempre cuatro variables en el objetivo de invertir un capital, trabajar con él y obtener unos beneficios sobre la inversión inicial. Estas cuatro variables o factores han sido la tecnología, el trabajo, el mercado y el entorno político. La tecnología equivale a las inversiones que se realizan: maquinarias, herramientas, locales. El trabajo significa la transformación de materias primas u otros inputs que tiene lugar en la empresa. El mercado es la realización de la venta de los bienes o servicios producidos. El entorno político equivale a las condiciones que la empresa ha de satisfacer para operar en determinado territorio como contrapartida a lo que recibe de la colectividad: impuestos y tasas, políticas sociales y medioambientales. Creo que se puede sostener que en teoría las empresas tienden a optar por un mercado o una tecnología poco flexibles -es decir, una tecnología que dure el máximo tiempo posible y un mercado que se mantenga o crezca pero sin sobresaltos- el máximo tiempo posible, sencillamente porque ello significa menos costes y más beneficios. En consecuencia, tienden a resolver las incertidumbres que necesariamente vendrán de la tecnología y del mercado -porque hay otras empresas en juego- utilizando flexiblemente el trabajo (más o menos, a menor o mayor costo, con mayor o menor rendimiento). Ello ha sido siempre así por tanto no cabe extrañarse demasiado de que las empresas vayan hoy buscando la máxima flexibilidad del trabajo, dado que es algo que han hecho siempre. La razón parece sencilla: si la flexibilización en el mercado o en la tecnología la realiza la empresa es ésta la que asume su coste, mientras que en la flexibilización del trabajo el coste -esfuerzo, rendimiento, salario y condiciones- lo suele asumir el trabajador.

Por el contrario, los trabajadores y los sindicatos han luchado contra la flexibilidad del trabajo, puesto que dicha flexibilidad implicaba asumir incertidumbres que podían ser dolorosas: de salario, de tiempo de trabajo, de condiciones de trabajo, de estabilidad. Con otras palabras la certidumbre de rendimiento que al empresario le proporciona la flexibilidad del trabajo tiene como contrapartida, para el trabajador, incertidumbre en sus condiciones de vida. Por tanto las empresas y sus trabajadores se han enfrentado desde siempre en torno a la cuestión de la flexibilidad del trabajo. En un momento histórico ese enfrentamiento asume una cierta regulación y con ella también se regula el grado de incertidumbre que el trabajador está dispuesto a asumir y el grado de certidumbre que el uso determinado de la fuerza de trabajo da al empresario. Es el momento de una mayor y más específica intervención del Estado y aquí tenemos la referencia al cuarto factor que he llamado contexto político. Desde la tercera década del siglo XX muchos Estados de los países con fuerte industrialización han contribuido a construir relaciones estables entre capital y trabajo basadas en la búsqueda de garantías mínimas para las dos 
partes: para la empresa garantía de estabilidad de costos para el trabajador garantía de estabilidad de empleo y de vida. El garante fundamental ha sido el Estado, a través de sus normas, tribunales, inspección. A cambio ha exigido a las empresas tasas e impuestos, salarios mínimos, condiciones de trabajo estándar. A los trabajadores y a sus sindicatos les ha exigido control del conflicto social y respeto, cuando este tiene lugar, al capital invertido por la empresa. El instrumento clave de todo este entramado de acuerdos tripartitos ha sido la negociación colectiva que, cuando se refiere al ámbito de país puede recibir el nombre de concertación. Pero detrás de ambas estrategias, de las empresas y de los trabajadores y sus representantes, hay el objetivo de conseguir la máxima certidumbre posible. Los años 60 y 70 son los de máxima certidumbre para los trabajadores. No es el momento de recordar ahora la historia de las relaciones laborales, aunque bastarían algunos ejemplos para verlo. En el Reino Unido los sindicatos logran implantar el closed shop y el control de los ascensos de categoría en muchas empresas. En Alemania entran en el Consejo de dirección de la empresa, con poco peso en ciertos casos, pero con mucho en otros. En Suecia los cambios tecnológicos mismos quedan sujetos a la negociación. En Italia la intervención de los comités de empresa en las condiciones de trabajo llega a ser determinante. Pero esta certidumbre para los trabajadores también se traduce en rendimiento para las empresas europeas, que tienen amplios mercados y gozan de amplio apoyo del estado, por su posición en la división mundial del trabajo.

La crisis de los años 80 cambia las cosas, porque las incertidumbres para las empresas comienzan a venir de las otras dos variables hasta entonces estabilizadas por la fuerza de los hechos, o por ciertas reglas de competitividad, o por la protección de los estados. Los mercados se hacen inestables, la tecnología se hace obsoleta a gran velocidad. Es entonces cuando las empresas necesitan poder superar incertidumbres de esos dos factores echando mano del incremento de la certidumbre en los otros dos en forma casi total. Por tanto se rompen las reglas de equilibrio hasta entonces vigentes en muchos países, comenzando las empresas a presionar para obtener cuotas más altas de flexibilidad del trabajo bien por la vía de los hechos sin más bien por la de la llamada des-regulación. La realidad es que el trabajo se convierte en el factor más fácil de manejar, más fácil de convertir en cierto, ante la tremenda incertidumbre de los demás factores. La intervención tradicional del Estado en las cuestiones laborales, ha dejado de ser una certidumbre, con normas e intervención, para convertirse en una incertidumbre por lo que exactamente lo que se le pide no es la no intervención, como frecuentemente se dice, sino la intervención de otra manera. La desregulación tiene ese significado, intervenir de otra manera. Pero también el deslizamiento desde la negociación colectiva hacia la negociación individual es un intento de alejar incertidumbres que provienen de pactos de mínimos garantizados o de condiciones iguales para la totalidad de los trabajä- 
dores de una empresa, un sector o un país. Ya no es una batalla para sustituir ciertas formas, digamos técnicas, de trabajar por otras de igual índole. Es una clara batalla en torno a los espacios de poder que la empresa y los trabajadores (o sus representantes) mantienen en lo que respecta al uso de la fuerza de trabajo. Cabe señalar, en esa línea, un retroceso del poder de los trabajadores que es más visible en el ámbito general que en el de las grandes empresas (Hyman, libro sindicatos). En estas, los representantes han conservado un cierto poder de negociación y, con ella, un control frente a posibles incertidumbres del trabajo. Pero esto se ha hecho posible a cambio de que dicha incertidumbre ha sido trasladada a la periferia del sistema de empresas -a las subcontratadas, a las pequeñas empresas que tienen que aceptar cualesquiera condiciones de las grandes- o a la "periferia laboral" de la misma empresa grande, es decir a los trabajadores que tienen contrato temporal o trabajan a tiempo parcial.

\section{Flexibilización del tiempo de trabajo}

Las formas de esta flexibilización son múltiples, pero quizá convendría detenerse en una que es la máxima manifestación de la flexibilidad del trabajo hoy, la flexibilidad del tiempo de trabajo. Desde el inicio de la revolución industrial el tiempo de trabajo ha estado en el centro de las luchas sociales de la relación laboral. Principalmente se trataba de la cantidad de días trabajados al año y de horas al día. La norma social del empleo en los países del estado del bienestar consigue también una estandarización del tiempo de trabajo: entre 40 y 45 horas a la semana, en muchos países repartidas en 5 días a la semana. También se registra un importante proceso de negociación que lleva a regular esas 8 o 9 horas al día: en horario continuado, en horario partido. Puede haber turnos pero con una cierta cadencia que se intenta acoplar a los otros tiempos de la vida: por ejemplo, sabiendo que el cambio de turno se dará cada mes o cada 2 semanas o bien que se mantendrá indefinidamente. El tiempo de trabajo, con todo, ha seguido siendo uno de los principales capítulos de la disputa entre trabajo y capital. Los trabajadores han intentado ir reduciendo al máximo dicho tiempo, sea en términos anuales que semanales, a favor de más tiempo para la vida privada. También han pugnado para que las variaciones estuviesen lo más reguladas posible por ley o por negociación. Por ejemplo, se han regulado los turnos o se ha conseguido que cambios de horario se deban avisar con varios días de antelación o bien se han regulado las horas extraordinarias. Todo ello, sobre la base de una jornada básicamente estable. Las empresas han intentado mantener jornadas lo más largas posible y también el máximo de control sobre las variantes de jornada. La fortaleza y la estabilidad de los modelos de relaciones laborales en buena medida se han basado sobre el mantenimiento de un objetivo muy claro: ocho horas diarias de trabajo en 5 días a la semana en un hora- 
rio preestablecido, siendo horas extras, turnos y variaciones horarias las menos posibles, negociadas y con contrapartidas. Y de hecho la tendencia que podemos observar en los países de estado de bienestar de los años 50 en adelante es la de reducción paulatina de las horas de trabajo anuales y semanales y la de estabilización de horarios.

Pero un cambio relevante tiene lugar a mediados de la década de los 90 , cuando se comienza a discutir en muchos países de la Unión Europea la reducción del tiempo de trabajo de 8 a 7 horas diarias. Los promotores del debate, los grandes sindicatos europeos, quieren conseguir dos objetivos con esta propuesta. El primero es un objetivo estratégico: relacionar la reducción del tiempo trabajado con el incremento del empleo. El segundo se refiere a dar un fuerte impulso a la mejora de las condiciones de vida, reduciendo el tiempo de trabajo que a lo largo de la actual crisis económica ha tenido un cierto incremento de la jornada de trabajo, particularmente en forma de horas extra (El Viejo Topo, 1998). Vamos a detenernos sobre el primero de dichos objetivos. Si la tecnología ha permitido ir incrementando la productividad del trabajo -argumentan los sindicatos- es lógico que ello tenga consecuencias no sólo a favor de la empresa, sino también de los trabajadores en un terreno en el que éstos están siendo claramente desfavorecidos: es decir, frenando la sangría de puestos de trabajo. En teoría, con una reducción semanal del trabajo en un $12.5 \%$ tiene que ser posible incrementar en forma notable los puestos de trabajo. El argumento de los empresarios para oponerse a esa medida es que sin una reducción proporcional del salario las cuentas en la empresa no salen. Pero muchos trabajadores no pueden asumir esa reducción salarial, a parte de que parece injusto, porque de ser así no se trataría sino de una subvención a nuevos puestos de trabajo realizada por los trabajadores mismos, no un aprovechamiento de la potencialidad de la tecnología. Con todo, el argumento de la tecnología no es muy sostenible en sectores intensivos en trabajo, como son muchos de los sectores de servicios.

Hay ejemplos muy citados como el de la empresa Volkswagen, que no son precisamente los más adecuados, pues sus trabajadores, entre los mejor pagados de Alemania, aceptan una reducción salarial y siguen estando entre los mejor pagados. El acuerdo en buena medida se explica porque dicha empresa tiene en Alemania una parte mayoritaria de capital público. La misma razón por la que en España el ejemplo ha cundido casi exclusivamente en instituciones públicas: ayuntamientos, universidades, diputaciones. También es cierto que en Francia se votan leyes (1998 y 2000) que introducen la jornada de 7 horas, con algunas excepciones en la empresa pequeña. No hay contrapartidas de reducción salarial, pero sí hay notables subvenciones gubernamentales a las empresas que crean nuevios puestós de trabajo, con lo cual el pago de los mismos no viene de los trabajadores, pero sí de los impuestos del conjunto de los ciudadanos. Se crean varios cientos de miles de puestos de trabajo, pero la oposición patronal y la llegada al poder de la dére- 
cha nuevamente en 2002 ponen en entredicho las leyes de reducción del tiempo de trabajo. Por otro lado, debería pasar un cierto tiempo para verificar si esos empleos subvencionados se han consolidado o no. Pero lo que resulta más llamativo es que a lo largo de los dos últimos años, y con la excepción de Francia por los debates políticos que origina la ley y por los problemas laborales a que da ocasión su aplicación, la cuestión de la reducción del tiempo de trabajo desaparece de la primera página de los debates y negociaciones entre patronal y sindicatos y de la agenda de los gobiernos o de la oposición. Es el caso de España, pero también el de Italia, el de Alemania y los de otros países en que este tema había sido muy discutido. La principal razón de ello es, a mi juicio, que la estrategia de flexibilidad del tiempo de trabajo se impone a la de reducción del tiempo de trabajo.

Así como la cuestión de la reducción del tiempo de trabajo había sido suscitada por los sindicatos con el fin de hacer frente a la crisis del empleo, la de flexibilidad del tiempo de trabajo ha tenido el protagonismo de las empresas, ofreciendo en contrapartida, allá donde hay representación laboral fuerte, garantías de mantenimiento del empleo o, a veces, incremento del mismo, sea fijo que temporal, aunque más el segundo que el primero. La flexibilidad del tiempo de trabajo tiene un aspecto material y otro social. Materialmente esa flexibilidad no necesariamente quiere decir trabajar más horas -aunque esto también puede ser-, sino distribuirlas diferentemente a lo largo del día, de la semana, del mes o del año. Pero más específicamente suele significar cambios de turnos a disponibilidad de la empresa, flexibilidad de entrada y, sobre todo, de salida. Probablemente el ideal para las empresas seria poder distribuir las, por ejemplo, 1700 horas anuales de trabajo más las posibles horas extras en la manera más flexible posible a lo largo del año. Por el contrario, dado que para los trabajadores poca flexibilidad suele significar más estabilidad en condiciones de vida y más posibilidades de disponer a su arbitrio del resto del tiempo de la vida diaria, tenderán a tiempos de trabajo lo más rígidos posible. Por supuesto que no siempre y en todos los casos, dado que quien trabaja a turnos puede querer flexibilidad para volver, lo más que pueda, a horarios más acordes con los tiempos del resto de su vida cotidiana. Las últimas frases nos abren la puerta a lo que se podría llamar el aspecto social de la flexibilidad del tiempo. Es decir, la distribución material podría parecer una mera fórmula técnica para resolver la distribución de las 1700 horas a través de todas las semanas del año laboral, pero no lo es. Y no lo es, por cuanto bajo una u otra forma de distribución laten objetivos e intereses de las partes que la convierten en un objeto de disputa, a veces muy dura. Así para los trabajadores la distribución del tiempo de trabajo puede condicionar fuertemente su vida fuera del trabajo, a veces situándolos en posiciones marginales respecto a cuestiones que les interesan mucho (Varios, 2001). Para las empresas la distribución del tiempo no sólo implica mayor o menor rendimiento, sino también mayor o menor control sobre su fuerza de trabajo, como ya hemos señalado anteriormente. 
Otros factores que cuentan en la flexibilidad del trabajo, además del tiempo, son desde el punto de vista del trabajador: el ritmo de trabajo, la intensificación del mismo, la calificación y otros. Todos ellos tienen que ver con la organización del trabajo que ha sido siempre el verdadero eje de la distribución del tiempo de trabajo, más rígido cuando así era lo adecuado -la típica cadena fordista- más flexible cuando las exigencias sociales, técnicas o del mercado lo requerían. También en estos casos, o genéricamente en la organización del trabajo, hay aspectos materiales y otros que son claramente sociales y que han sido estudiados muchas veces (Coriat,. 1992). Trabajar a un ritmo o a otro, asumir movilidad funcional o geográfi$\mathrm{ca}$, intensificar el trabajo han supuesto, en el fondo, unas u otras condiciones de vida. Otra cosa es que, con la disminución histórica de la jornada de trabajo, las condiciones de vida que se derivan del trabajo pueden haber estado menos condicionadas por la actividad laboral en el conjunto de la vida cotidiana de muchos, precisamente porque otras actividades y otros objetivos han ganado terreno.

\section{FleXibiLIDAD DEL EMPLEO}

Hasta ahora hemos estudiado las estrategias de flexibilidad del trabajo dentro de las empresas. Pero estas están interesadas no sólo en disponer de la fuerza de trabajo en manera adecuada a sus necesidades dentro de sí mismas, sino también en que los trabajadores ingresen en ellas con unas características -veremos luego que éstas implican también disponibilidades- marcadas por el mercado de trabajo. Ello es posible a través de lo que se llama flexibilidad del mercado de trabajo. La flexibilidad del mercado de trabajo quiere decir básicamente flexibilidad del costo del trabajo (Recio, 1988) pero no sólo éso. Algo que en países con escasas normas y escasa o nula representación colectiva de los trabajadores, se resolvería en forma bastante simple en una fijación del salario entre empresa y trabajador, que podría variar en cada momento, requiere otros mecanismos en los países en que hay algún tipo de regulación. En general el mecanismo utilizado en estos casos es el del contrato. Flexibilidad del mercado de trabajo quiere decir que existan y se puedan escoger varios tipos de contratos para la compraventa del factor trabajo. En términos globales solemos diferenciar entre contratos indefinidos y contratos temporales, con varias modalidades en cada uno de ellos, en particular en el segundo tipo. Pero como esa flexibilidad del mercado de trabajo da origen a formas bastante diferenciadas de relación entre empresa y trabajador y es usual hablar en este caso de flexibilidad del empleo.

El análisis sociológico de la flexibilidad del empleo no puede contentarse con su aspecto más evidente y más material, es decir, con el recuento del número de empleos indefinidos y temporales, preguntándose a renglón seguido por las causas y las condiciones de cada uno de esos dos tipos. Hay, al menos, otras dos cuestio- 
nes, no tan evidentes pero quizá mucho más importantes, que definen el verdadero significado social de la flexibilidad del empleo hoy día. La primera es la escasa consistencia de la estabilidad contractual, la segunda la enorme variabilidad de la inestabilidad (que consigue que ciertos empleos inestables hasta puedan parecer bastante estables en comparación con otros, lo que implica una enorme división de intereses entre los trabajadores). Se está acabando el "empleo para toda la vida" con la excepción de los funcionarios, por ahora- puesto que muchos empleos formalmente indefinidos o estables son en realidad muy inestables: la empresa puede deslocalizarse, cerrar, realizar reducción de plantilla, despedir aunque sea con indemnización. Se puede argumentar contra esta afirmación diciendo que tampoco en el pasado el contrato indefinido era literalmente para siempre. Pero la realidad es que en los países de la regulación, una buena parte de ésta pretendía justamente la mayor durabilidad posible del contrato. Había diversas formas de lograrlo: hacer caro el despido, construir una fuerte estructura pública de lucha contra el desempleo. Sobre todo existía un sistema de relaciones laborales fuertemente consolidado cuyo punto de equilibrio era ni más ni menos que la estabilidad del empleo a tiempo completo (aunque básicamente para los hombres), pero todas esas garantías y plataformas sociales se debilitan. En buena parte, la "inestabilidad" del empleo formalmente estable se debe a la existencia de una fuerte tasa de paro y a un creciente porcentaje de empleo formalmente inestable. En efecto, cuando en el mercado de trabajo hay mucha mano de obra dispuesta a trabajar, aunque sea en malas condiciones, los costes del despido o de la reducción de plantillas se relativizan a medio plazo, porque a las empresas les puede interesar despedir sin causa, aunque tengan que pagar el despido, y contratar trabajadores a menor coste.

Por otro lado tenemos la enorme variabilidad del trabajo inestable. Es inestabilidad por el tipo de contrato, inestabilidad por el tipo de empresa que contrata, inestabilidad derivada del sector -casos de la agricultura o la hostelería o el espectáculo- o bien de las de las horas trabajadas, etc. De manera que el contrato de obra y servicio puede parecer un privilegio en comparación con muchos que son de un año o un mes o una semana. O bien un contrato temporal en banca o el sector energético puede ser un sueño en relación con el mismo tipo de contrato en la construcción o en la agricultura. Es decir, hay empleos inestables que son mucho más inestables que otros y algunos formalmente inestables que son más estables que los que formalmente tienen esa característica contractual. Lo que se está produciendo es una enorme fragmentación de los trabajadores inestables, justamente en razón de la mayor o menor consistencia, o inconsistencia, de dicha inestabilidad. El resultado subjetivo, para los trabajadores, de esa doble posible inestabilidad, es el crecimiento espectacular del riesgo de caer en situacionęs más precarias, es decir, la sensación de inseguridad en el trabajo. En ambos aspectos vuelven a entrar en juego los significados profundos de la flexibilidad laboral-que examinaré 
en el apartado siguiente-, la lucha contra la incertidumbre del trabajo y la presión por la disponibilidad del mismo.

En todos los tipos de contratos no indefinidos a tiempo completo parece resuelto el problema que tienen las empresas respecto a la materialidad de la cuestión, en el sentido de que se utilizaría la fuerza de trabajo únicamente en el periodo necesario y al mínimo coste necesario (hay que recordar que se eliminan costes de antigüedad y otros pluses que con frecuencia tienen los trabajadores fijos, como pagos por objetivos, posibles terceras pagas, etc.). Es decir, el trabajo se emplea como factor cierto y se dispone de él en el tiempo estrictamente necesario. Pero este tipo de contrato y de relación no garantiza el máximo rendimiento posible; por cuanto éste depende en gran parte de actitudes mentales, por las cuales uno puede dar lo máximo de sí mismo en el trabajo o, por el contrario, dar lo mínimo para pasar. Probablemente ésta es una de las razones por las cuales han tenido un desarrollo tan importante las políticas de Recursos Humanos. Justamente lo que buscan estas políticas es la implicación de los trabajadores en los objetivos de la empresa. Y cabe decir que en las últimas décadas estas políticas han tenido bastante éxito, por dos motivos principalmente, a mi entender. El primero ha sido el debilitamiento de la identificación colectiva y el consiguiente incremento de los sentimientos individualistas de los trabajadores; ello ha facilitado el tejido de una cierta identificación con la empresa, en alguna de sus dimensiones: el producto, los métodos de trabajo, la formación. El segundo ha sido la crisis del empleo, debido a la cual muchos trabajadores han entendido que pelear a fondo por la competitividad de la empresa era defender el propio puesto de trabajo (aunque a la mínima crisis algunos hubiesen de constatar que no era asî) o bien no hacerlo era arriesgarse al despido dada la inseguridad de su empleo estable. Pero justamente esas políticas de Recursos Humanos han sido menos adecuadas con los trabajadores de empleo flexible (formalmente, los no estables), puesto que al no ser posible la expectativa razonable de continuidad en el empleo, es decir en la empresa, tiene poco sentido para los trabajadores no estables, es decir para los "Recursos Humanos prescindibles", implicarse con todas las fuerzas en el trabajo y responsabilizarse personalmente por la competitividad de la empresa. Esa es la razón por la que, de hecho, las políticas de personal aplicadas a este tipo de trabajadores no son tanto de implicación como de "coacción", aunque ésta pueda tener modos suaves, que en general tienen que ver con la alternativa entre renovar el contrato temporal o no hacerlo, lo que resulta bastante importante en un panorama de crisis del empleo.

En consecuencia, la flexibilidad del empleo ha dado a la empresa ciertas ventajas en un terreno que podríamos calificar más bien de técnico. Es decir, por lo que respecta a poder calcular con mayor precisión el trabajo estrictamente necesario, en el momento necesario, lo que podríamos llamar la "disponibilidad material". Pero las ventajas han sido menores en lo que respecta a la "disponibilidad mental". 
de la fuerza de trabajo, dado que ésta no siempre ha podido ser implicada a pleno rendimiento, más allá de las horas formalmente pasadas en la empresa. Es más, parece difícil que muchos trabajadores en riesgo de perder el puesto de trabajo tengan interés en implicarse en las políticas o los objetivos de la empresa, es decir, manifestarse disponibles. En estos casos hay una disponibilidad material, pero no una disponibilidad mental. Es la razón por la cual las políticas de recursos humanos suelen dejar de lado a los trabajadores que están en precario.

6. El SIGNIFICADO DE LA FLEXIBILIDAD LABORAL: CERTIDLiMBRE VERSUS INCERTIDUMBRE, DISPONIBIIDAD VERSUS AUTONOMLA.

El significado de la flexibilidad no es algo que sea derivable técnicamente de la materialidad del fenómeno que hemos descrito, puesto que, como ya se ha dicho, este tiene aspectos también socio-políticos. Son éstos los que hacen que la flexibilidad no sea un fenómeno de lectura homogénea, sino que pueda ser asumido desde puntos de vista diversos, a veces contradictorios. Para las empresas flexibilidad del trabajo y del empleo -y más la última que la primera- significa certidumbre y posibilidad de disponer más y mejor de la fuerza de trabajo. Para los trabajadores significa incertidumbre -excepto en los casos en los que las formas de flexibilidad están negociadas y pactadas o normadas, aunque siempre con el riesgo de que alguien pueda no respetar lo acordado- y mayor disponibilidad hacia la empresa. Lo primero implica riesgo de cara al futuro, lo segundo supone tener menos disponibilidad para ordenar el tiempo de la vida privada.

Los estudiosos suelen diferenciar dos tipos de flexibilidad laboral, a una la llaman flexibilidad interna y a la otra flexibilidad externa. Están hablando, en ambos casos, de la utilización del trabajo por las empresas más bien que de decisiones autónomas de los trabajadores en cuyo caso deberíamos hablar no de flexibilidad, sino de autonomía laboral. Para algunos trabajadores, probablemente pocos, trabajar sin contrato o bien con contrato a tiempo parcial o bien con máximas posibilidades de movilidad, la flexibilidad puede ser buena puesto que refuerza su autonomía y las consecuencias positivas de la misma (mejoras de salario, condiciones mejores de trabajo, buena relación entre trabajo y cualificación, etc). Para el resto de trabajadores la flexibilidad tiene, o puede tener en algún momento, consecuencias negativas.

En ambos casos, flexibilidad interna y externa, la empresa va buscando dos objetivos en su relación con el trabajo: el obtener una posición de máxima certidumbre y mínimo riesgo y el conseguir una actitud de máxima disponibilidad material de esos trabajadores. Ha habido en los últimos años un intenso debate sobre regulación y desregulación y dicho debate, con frecuencia, se ha referido a la intervención del Estado o, lo que es lo mismo, a las relaciones entre economía y políti- 
ca. Por ello la regulación se ha visto como un conjunto de normas y leyes que establecen las pautas de uñ interés común por encima de los intereses particulares de los dos protagonistas de la producción, el capital y el trabajo, siendo ese interés común el equilibrio o la cohesión social. Pero si entramos más a fondo en el carácter de la relación entre capital y trabajo percibimos que lo que ha hecho la regulación ha sido introducir condiciones y requisitos en la relación libre entre ambos protagonistas. ¿Por qué? Sencillamente porque se trata de una relación desequilibrada en la que hay uno que es siempre más fuerte que el otro. Por ello la regulación ha recortado dicha libertad -que básicamente lo era para uno de los contendientesen aras a poder mantener la sociedad ( la cohesión social). Es, por tanto, comprensible que los empresarios hayan sido siempre contrarios -en mayor o menor grado según las sensibilidades personales de cada cual y los resultados de las empresas- a esa intromisión del estado y de la sociedad, no por otra razón sino porque la regulación ponía límites a las meras relaciones de mercado, es decir, a su libertad de actuación.

Pero si las cosas son como estoy indicando, cabe pensar que la desregulación no ha de significar necesariamente eliminación o disminución de reglas y normas, sino incremento de la libertad de actuación que se había recortado. Por ello precisamente lo que está pasando efectivamente no es que disminuyen las reglas sino que cambian las reglas. Cambian las reglas y normas de la contratación, del despido, de la jornada, de la protección al desempleo, reforzándose la libre contratación, etc. Con el cambio de reglas, que en general va en la línea de abandonar aplicaciones generales -las llamadas "erga omnes"- para definir grupos o colectivos más circunscritos y particulares como sujetos de garantías, se debilita una situación que podríamos llamar de garantías mínimas o los mecanismos de estándares mínimos para todos, y vuelve a primer término la fuerza real de las partes. Por esta razón las nuevas normas suelen tener poco efecto desregulador allí donde hay sindicatos o comités de empresa fuertes, pero lo tiene y muy notable donde no se da esta circunstancia, es decir, donde la sindicación es débil o dividida. Por el contrario, desregulación ha significado para los empresarios incremento de libertad para aplicar los criterios o las formas de trabajar que mejor pueden satisfacer a sus objetivos. Por tanto en el grado en el que esta libertad empresarial se está incrementando -lo que puede ser en la empresa central o en las que dependen de la mismapodemos hablar de triunfo de la desregulación.

Pero el objetivo de incrementar la libertad de actuación de la empresa va unido al de obtener la máxima disponibilidad material de los trabajadores. Esa disponibilidad significa básicamente realizar la actividad laboral coincidiendo con los momentos en que ésta puede ser de máximo rendimiento para la empresa. Ese es el significado del trabajo a tiempo parcial, de las horas extras, del contrato temporal. Parece obvio que esa disponibilidad queda mejor garantizada si hay normas que la 
establecen, más que si es sólo resultado de una correlación de fuerzas en la empresa que, en otra coyuntura, podría cambiar. Por tanto, la nueva regulación, resultado de una mayor influencia política del capital y de una menor influencia política del trabajo, no sólo amplía el grado de libertad de aquel, sino que convierte a éste en más disponible en todos los aspectos, lo que se traduce en normativas más garantistas.

Es una disponibilidad que sobre todo entra en los aspectos cualitativos de la relación capital trabajo. Permite aprovechar los tiempos poniendo de relieve aquellos que son de mayor interés para la empresa -por ejemplo, los de coyunturas económicas positivas- o aquellos en los que el trabajador puede estar más descansado. Permite aprovechar los conocimientos del trabajador no sólo en una tarea, sino quizá en varias tareas. Posibilita pagar cara o barata la fuerza de trabajo según rendimientos medidos mucho más en detalle que si hablamos de salarios estándar o muy pautados.

Incremento de la libertad de la empresa en el uso de la fuerza de trabajo y aumento de la disponibilidad del trabajo se convierten en factores claves para resolver incertidumbres en el panorama empresarial o bien son certidumbres que permiten hacer frente a la incertidumbre que puede provenir del mercado y de la tecnología.

\section{BIBLIOGRAFIA:}

- Aglietta M. (1987) A Theory of capitalist regulation. The USA experience. Verso, London.

- Alonso L.E. (2000) Trabajo y posmodernidad: el empleo débil. Fundamentos, Madrid.

- Alós R., Míguelez F., Recio A.(1988) El trabajo precario en el comercio. Ceres, Barcelona.

- Bilbao A. (1993) Obreros y ciudadanos. Trotta, Madrid.

- Boyer R. (1986) La flexibilidad del trabajo en Europa. MTSS, Madrid.

- Burawoy M. (1989) El consentimiento en la producción. Los cambios del proceso productivo en el capital monopolista. MTTS, Madrid.

- Castel R. (1995) Les métamorphoses de la question sociale. Une chronique du salariat. Fayard, Paris.

- Castells M.(1997) La era de la información. Vol 1, Economía, sociedad y cultura. Alianza, Madrid..

- Coriat B.(1992) El taller y el robot. S.XXI, México-Madrid.

- Harrison B. (1997) La empresa que viene. Paidós, Barcelona. 
- Kern H., Schumann M. (1988) El fin de la división del trabajo. Racionalización en la producción industrial. MTSS, Madrid.

- Martin A.(1995) Flexibilidad y relaciones laborales. Estrategias empresariales y acción sindical. CES, Madrid.

- Miguelez F., Prieto C. (1991) Las relaciones laborales en España. S.XXI, Madrid.

- Miguélez F., Prieto C. (1999) Las relaciones de empleo en España. SXXI, Madrid.

- Miguélez F., Prieto C (2002) "Crisis del empleo y cohesión social", Cuadernos de relaciones laborales $\mathrm{N}^{\circ} 20$, Madrid.

- Offe C. (1991) La sociedad del trabajo, Alianza, Madrid.

- Piore M., Sabel Ch. (1990) La segunda ruptura industrial. Alianza, Madrid.

- Polanyi K.(1989) La gran transformación. La Piqueta, Madrid.

- Pollert A. (1991) Farewell to Flexibility? Basil Blackwell, London.

- Prieto C.(2002) "Sobre la norma social de empleo" Sistema No168-169. Madrid

- Recio A. (1988) "Flexibilidad, eficiencia y desigualdad", Sociologia del Trabajo $\mathrm{n}^{\circ} 4$.

- QUIT (1997). Economia, trabajo y empresa. CES, Madrid.

- QUIT. (2002) Las implicaciones del reparto del trabajo sobre el empleo y la vida cotidiana. Investigación patrocinada por la Dgicyt. UAB. Informe final.

- Varios (2001) Temps i ciutat. Consejo Económico Social de Barcelona, Barcelona.

- Viejo Topo (1998) El libro de las 35 boras. El Viejo Topo, Madrid

- Watson T. (1994) Trabajo y Sociedad. Hacer, Barcelona. 